\title{
A Blade Surface Flatness Measuring System Based on Laser Displacement Sensor for Knives and Scissors
}

\author{
Shi Wenqing ${ }^{a}$, Yang Ziyuan ${ }^{b}$, Liao Weifeng ${ }^{c}$, Deng Yubin ${ }^{d}$ and Zhang Lixia ${ }^{e}$ \\ College of Science, Guangdong Ocean University, Zhanjiang, Guangdong, China, 524088

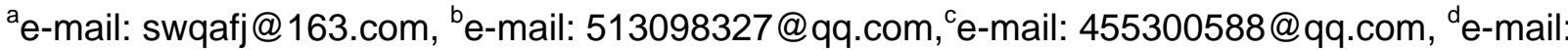 \\ 495600315@qq.com, ee-mail: 757552638@qq.com
}

Keywords: measuring system; laser displacement sensor; blade edge surface flatness; knives and scissors.

\begin{abstract}
In order to measure the blade surface flatness, which has a serious influence on the quality and its stability of knives and scissors quality, a blade surface flatness measuring system based on laser displacement sensor is established. This system has many advantages such as a high precision $(0.001 \mathrm{~mm})$, non-contact, convenient, simple operation, and so on. The system is very helpful to improve the product qualities of knives and scissors.
\end{abstract}

\section{Introduction}

The blade is the core of a knife or a pair of scissors, it plays an important role in cutting. How to make the blade is one of the key technology that influence the qualities of a knife or a pair of scissors. The factors which influence the blade qualities include the blade materials, edge angle, surface flatness, and so on.

As we know, the blade material is the most important factor in making a good knife or a pair of scissors. Using different materials will have entirely different results in the quality of knives or scissors.

There are different knives and scissors in quality even though using the same material. The blade edge angle is another important factor. The angle of the knife edge produced by the sharpening and honing operation is called blade edge angle. Different blade edge angles have different performance in cutting ${ }^{[1]}$. If a knife is often used in hard object cutting, the blade edge angle should be less acute; but for the knife often used in cutting soft tissue, the blade edge angle should be more acute ${ }^{[2,3]}$. Knife blade is made in different edge angles for various uses. For example, the common kitchen knife blade edge angle is generally about 50 degrees, and the fruit knife blade edge angle is generally about 30 degrees. The same kind of knives or scissors should have almost the same blade edge angle. Those knives and scissors, whose blade edge angle goes beyond the scope of certain value, will be considered as substandard products ${ }^{[4,5]}$.

Making blade is a crucial step in the processes of manufacturing knives or scissors. The process of making blade determines the sharpness and sharpness retentivity of knife or scissors. There is a complete industrial chain for knives and scissors in Yangjiang city, and the products of knives and scissors that made in Yangjiang occupy nearly 80\% market share in china. But hand making may be the most common process of making blade in Yangjiang. Hand making will have very great random in making blade. The blade edge angle is not very uniform and the blade surface flatness is not very flat after making blade by hand, and there is a lack of instrument or equipment for measuring the knife blade surface flatness of knives or scissors promptly.

In order to improve product quality of knives and scissors, a set of evaluating methods and system should be set up. So it is very important to project the method for measuring the blade surface flatness of knives or scissors rapidly.

\section{Principle of laser displacement sensor}


The laser displacement sensor is an optoelectronic device for measuring distances accurately. It composes of laser, detectors, measurement circuit and so on. It uses high speed non-contact laser gages to measure products in process. The principle of operation operates on the basis of non-contact comparative phase measurements with amplitude modulation. It uses a laser diode as its source with a class 2 rating for plant safety. Aiming alignment is easily achieved with the help of the red laser beam. The measuring range of the system based on the laser displacement sensor is between 0.05 to $50 \mathrm{~mm}$ (this measuring range is far beyond the blade thickness of common knives or scissors). The measuring principle of the laser displacement sensor is based on the comprehensive knowledge of optical triangle method ${ }^{[6]}$. It is shown in figure 1.

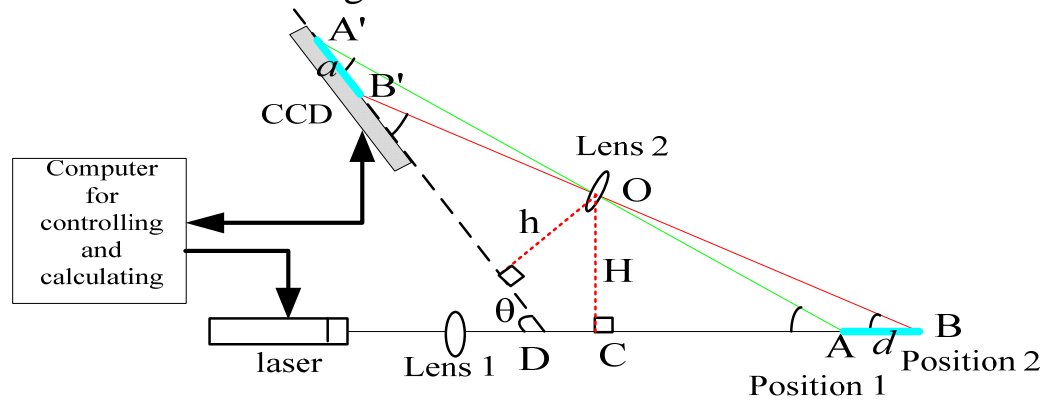

Fig. 1 The measuring principle of laser displacement sensor by optical triangle method

Laser beam, which is focused by lens 1, is reflected from the blade surface to the CCD (charge coupled device) to be detected (position 1 or 2). The information from the CCD is transferred to the computer to be treated. All these processes are controlled harmoniously by the computer.

We can draw the conclusion from figure 1 that different imaging points in CCD (shown in figure 1 as $A^{\prime}$ or $B^{\prime}$ ) come from different reflection positions (position $A$ or $B$ ). Suppose the length of $A B$ is $d$, the length of $A^{\prime} B^{\prime}$ is $a$, and the angle of $\angle A O B$ is $\varphi$. It is proved that there is a one-to-one correspondence between $a$ and $d$. For given $h, H 、 \theta, d$, we can obtain the follow relationships from the geometric relation in figure 1 :

$$
a=\frac{h}{H} \cdot \frac{\angle O A C-\varphi}{\theta-\angle O A C-\varphi} \cdot d
$$

From Eq. (1) above, we can obtain the value of $a$, which means the blade surface flatness variation of knives or scissors.

\section{Principle of the blade surface flatness measuring system}

The blade surface flatness measuring system based on laser displacement sensor for knives or scissors is organized as follow:

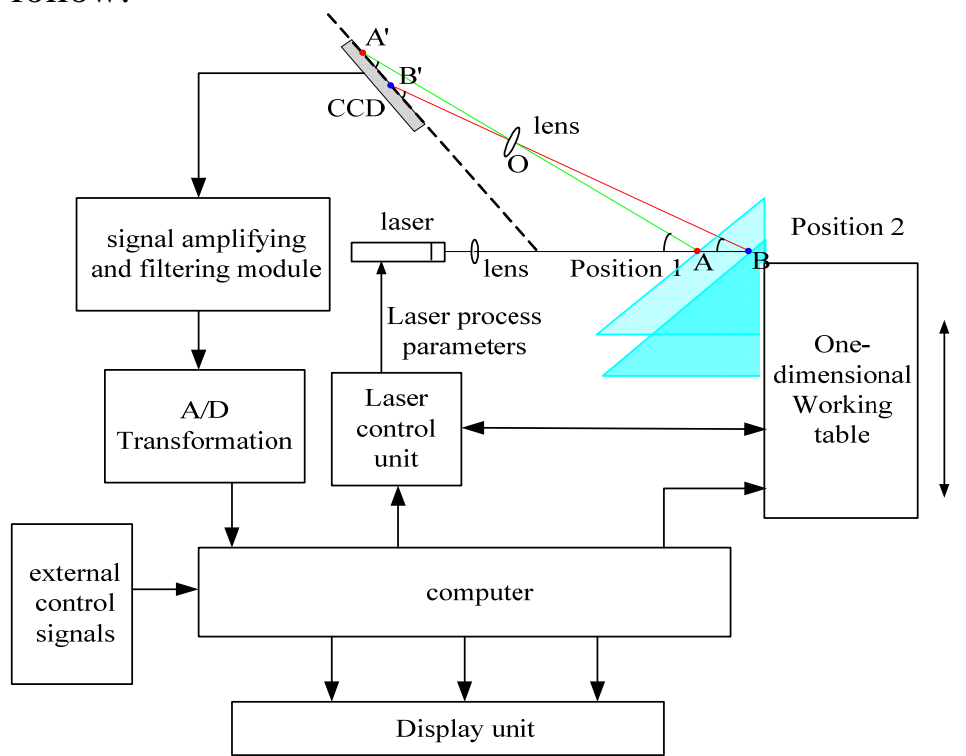

Fig. 2 the principle of the blade surface flatness measuring system based on laser displacement sensor 
Firstly, the knife or the scissors to be measured should be fixed on the working table of the blade surface flatness measuring system. Secondly, laser beam irradiates the blade surface of knife or scissors and reflects from it to CCD. Thirdly, the signal from CCD is amplified, filtered and transformed from analog to digital signal. Finally, the computer treats, calculates the data and displays the blade surface flatness on the screen. All these steps are controlled by the computer.

The relative movement occurs between the laser displacement sensor and the working table. The working table moves toward or away from the laser displacement sensor. The movement of the working table is driven by a computer-controlled high performance stepping motor system. Moreover, both the working table and the laser displacement sensor are controlled by the computer ${ }^{[7]}$. The structure scheme of the blade surface flatness measuring system is shown in figure 3.

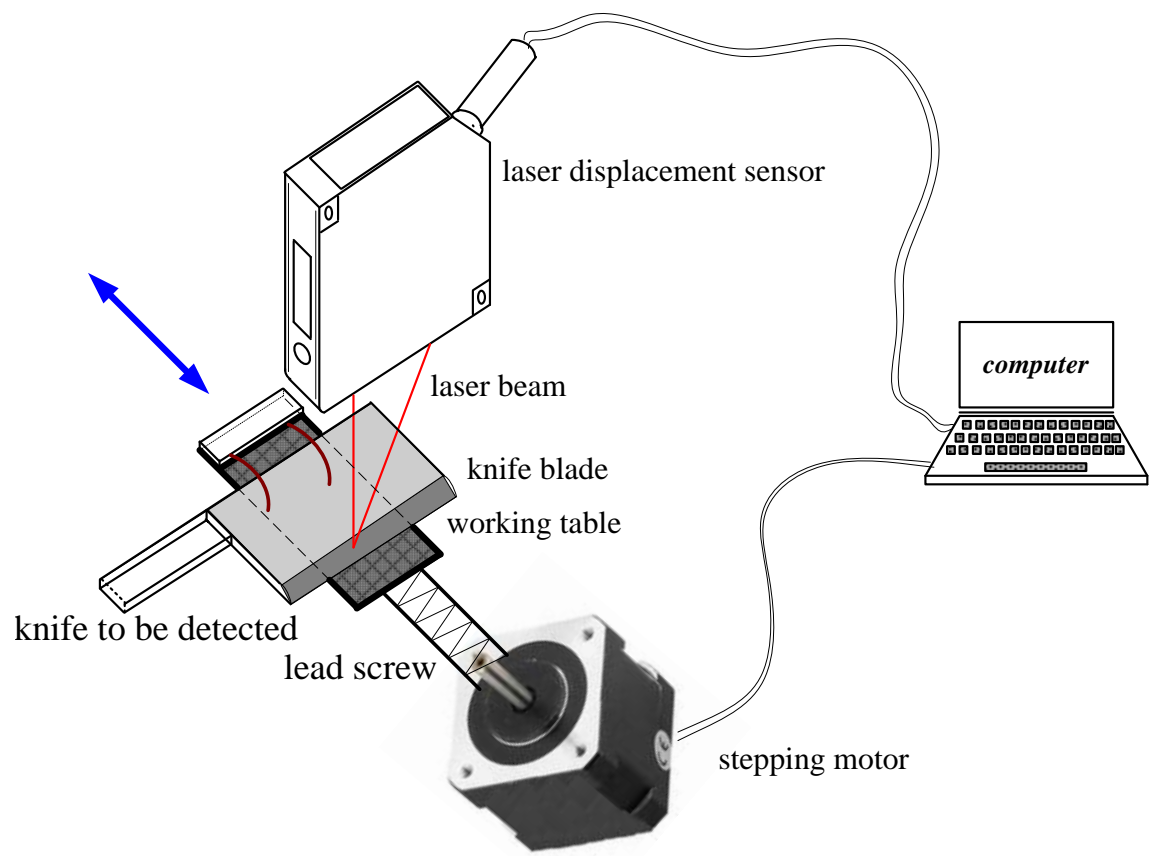

Fig. 3 the structure scheme of the blade surface flatness measuring system based on laser displacement sensor

The working table, on which the knife or scissors fixed, moves in one-dimensional direction with a lead screw drive. The lead screw is driven by a stepping motor. If the computer gives a signal to the stepping motor, the stepping motor will rotate a certain angle, and the working table will move a displacement. The displacement which lead screw moves is in one-to-one correspondence with the angle which stepping motor rotates. Due to the small thread pitch of the precision lead screw, the working table can move very small displacement accurately by means of the stepping motor rotation. The laser displacement sensor system sends laser beam and receives the signal reflected from the knife blade every displacement of the working table. With the rotation of the stepping motor, the working table moves, laser beam from the laser displacement sensor irradiates different positions of the knife blade. The signal which represents different thickness of the blade is obtained. Finally, the blade surface flatness trend of longitudinal direction displayed on the screen after treating and calculating by the computer.

\section{Conclusion}

The knife blade surface flatness is one of the most important factors which influence the sharpness and sharpness retentivity of knives and scissors. A blade surface flatness measuring system based on laser displacement sensor for knives and scissors is presented in this paper. This system has many advantages such as a high precision $(0.001 \mathrm{~mm})$, non-contact, convenient, simple operation, safe and reliable, and so on. This is very helpful to improve the product qualities of knives or scissors, and can promote the knives and scissors industrial development. The surface flatness measuring system also can be widely used in other industrial fields, especially in real-time detection of work piece surface. 


\section{Acknowledgments}

The work was supported by the Innovation and Venture Foundation for Guangdong Ocean University Students (CYXL12015).

\section{References}

[1] John D. Verhoeven, Alfred H. Pendray and Howard F. Clark. Wear. 2008, 265:1093.

[2] Raymond W. Mc Gorrya, Peter C. Dowdb and Patrick G. Dempsey. Applied Ergonomics. 2005, 36:71.

[3] Feng Yujun, Xiong Zhengye, Li Yongqiang, Huang Cunyou, Shi Wenqing and Chen Jinmin. Advanced Materials Research. 2011, 308-310:469-472.

[4] Shi Wenqing, Feng Yujun, Wang Wenhua, Li Yongqiang, Huang Cunyou, Xiong Zhengye, Tan Zhongming and Liang Su. Advanced Materials Research. 2011, 317-319:1016-1019.

[5] An Fenju, Feng Yujun, Tan Zhongming, Shi Wenqing, Xiong Zhengye, Li Yongqiang, Huang Cunyou and Wang Wenhua. Advanced Materials Research. 2011,339:80-83.

[6] Zhu Wanbin, Zhong Jun and Mo Renyu. Transducer and Microsystem Technologies. 2010, 29(6):131-133.

[7] Yuan Jiang, Qiu Zixue and Shao Jianxin. Chinese Journal of Scientific Instrument. 2010, 31(11):2488-2493. 